\title{
Set Point Control for Charging of Electric Vehicles on the Distribution Network
}

\author{
Colin Harris, Ivana Dusparic, Edgar Galván-López, \\ Andrei Marinescu, Vinny Cahill and Siobhán Clarke \\ School of Computer Science and Statistics \\ University of Dublin, Trinity College \\ Dublin, Ireland \\ \{colin.harris, ivana.dusparic, edgar.galvan, marinesa, vinny.cahill, siobhan.clarke\}@ scss.tcd.ie
}

\begin{abstract}
Many countries envisage a future where renewable electricity will be the predominant energy source. For example, Ireland's smart grid roadmap has targets of $40 \%$ of electricity from renewables by 2020 and $80 \%$ by 2050 . To achieve these targets will require new ways of operating the grid. We propose that there will be two types of demand, a load which can be influenced by dynamic pricing and a more tightly controlled flexible load that can be used to shape the aggregate demand. Key examples of this flexible load are electric vehicles (EVs), electric storage heating and hot water heating. This paper explores two algorithms that implement tight set point control for a set of EVs on a distribution feeder line. The first algorithm uses a variable power charger for charging the EVs and the second algorithm shows that it is possible to achieve similar results with a much simpler on-off charger.
\end{abstract}

Index Terms-- demand side management; electric vehicle charging; set point control; smart grid

\section{INTRODUCTION}

Many countries envisage a future where renewable electricity is the predominant energy source as we attempt to break away from diminishing fossil based fuels. For example, Ireland's smart grid roadmap has targets of $40 \%$ of electricity from renewables by 2020 and $80 \%$ by 2050 [1]. Renewable supply will come from wind, wave, hydropower and biomass and there will be significant increase in demand mainly due to the electrification of vehicles and heating appliances.

Achieving these targets will require new engineering solutions and new ways of operating the grid. Demand side management will be key in this new smart grid, enabling energy efficiency, control of demand to match intermittent renewable supply and control of demand to avoid congestion and overloading. For example, the significant increase in demand will require the distribution network to be significantly upgraded unless an approach to coordinating the demand can be engineered.

Current "manual" demand side management approaches do not influence the shape of the demand significantly. For example, a smart meter trial conducted in Ireland (2009-2010) on 6,000 residential houses tested the use of in home displays and dynamic pricing [2]. The intention was to both inform the customers of their electrical energy consumption and influence their use of electrical appliances through dynamic pricing. The trial showed a relatively modest saving in energy (1-2\%) and a slightly better shift in peak demand (9\%).

Fig. 1 shows an example row of houses being fed by a single transformer. We propose that in a future smart grid scenario there will be two types of demand, a load which can be influenced by dynamic pricing (termed base load) and a more tightly controlled flexible load that can be used to shape the overall aggregate demand.

Fig. 2 shows this aggregated demand at the transformer being controlled to a current set point level. This is made up from the variable base load and the flexible load being controlled to this given set point.

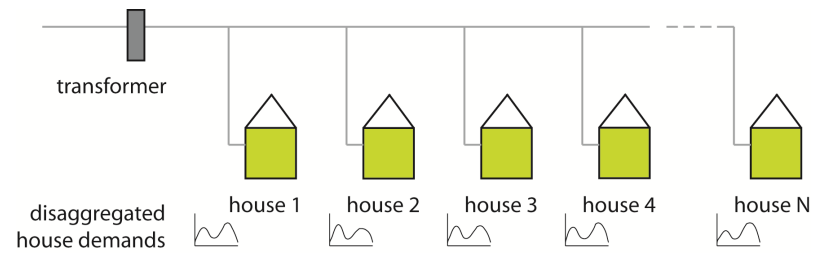

Figure 1. A row of houses being fed by a transformer.

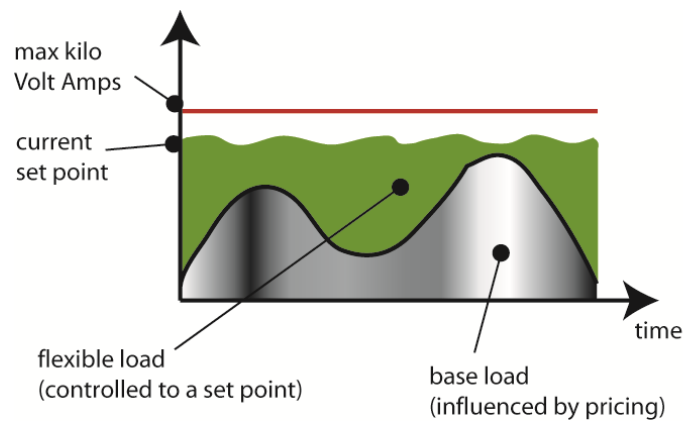

Figure 2. Aggregated demand controlled to a set point. 
Flexible load consists of appliances that have significant energy consumption and have storage so they can accept energy from the grid flexibly and deliver their service to the customer when they require it. Key examples of this flexible load are electric vehicles (EVs), electric storage heating and electric hot water heating.

This paper explores two algorithms that implement tight set point control for a set of EVs on a distribution feeder line. The test scenario is based around charging of EVs. The objective is to fairly charge each of the EVs as quickly as possible without overloading the distribution transformer. This maximises the efficient use of the grid infrastructure, while maintaining user utility of the EV.

We first present some related work in the area in Section II. Then Section III presents the design of the two algorithms, Section IV presents the experimentation and results, and finally Section $\mathrm{V}$ gives conclusions and future work.

\section{RELATED WORK}

There have been many papers that address the problem of charging of EVs on a constrained distribution grid [3, 4, 5, 6]. Here we look in detail at three approaches, their aim being to achieve fair charging of the EVs, as quickly as possible and without overloading the distribution grid components. These solutions give insights into the design of the algorithms that this paper develops.

\section{A. Linear Programming}

Richardson et al. [7] present an optimal centralised solution based on linear programming that solves the given objective function:

$$
\mathrm{F}=\sum_{\{=1}^{N} p_{\delta V_{l}} x_{l}
$$

where $\mathrm{N}$ is the number of EVs on the grid, $P_{\delta y_{l}}$ is the power allocated to the $i^{i h} \mathrm{EV}$ charger and $x_{i}$ is a weighting function. The objective function is maximised at each 15 minute timestep to fairly calculate the maximum allowable power for each of the EVs, while keeping within the network constraint limits. The key constraints are (i) not exceeding the transformer power limit, (ii) staying within voltage limits at the house connections, and (iii) ensuring smooth ramping up and down of the EV charger. They state that sharp changes in EV charging power are bad for the battery and reduce its lifetime.

The algorithm is centralised at the transformer and it requires two-way communications to retrieve data from the EV chargers and send control signals to the EV chargers. The solution solves the problem in theory but there are a number of practical drawbacks. Firstly, the two-way communications are expensive to implement and can suffer from network delays and reliability issues. The linear programming solver is computationally intensive and takes a significant time to calculate the power values. Also, the centralised nature of the algorithm makes it not very scalable. Furthermore, we believe that the time step of the controller needs to be smaller (i.e. at least once per minute), as the network demand could vary significantly within the 15 minute interval causing the network to overload. This more frequent time step will make the algorithm even more computationally intensive and subject to delays. Also, the electrical network needs to be modelled in a simulator in order to calculate the network sensitivities for the calculations. This would be very labour intensive in practice.

The EV charger used in the algorithm has a variable charge rate that can modulate the charge power from 0 to $100 \%$ as needed. This type of charger requires power electronics to modulate the power and can cause noise and harmonics which deteriorate the power quality of the network. Filters are then needed in the EV charger to reduce its noise emissions. A simpler, on-off type of EV charger does not cause these noise emissions and is a much cheaper solution.

Ideally, we would like to find a more distributed, scalable algorithm that can use the simpler on-off type charger and still come close to the theoretical performance of the centralised linear programming approach.

\section{B. AIMD Framework}

Studli et al. [8] present an approach based on a solution to congestion control in the internet, using additive increase, multiplicative decrease (AIMD) algorithms. As shown in Fig. 3 , the solution is simple to implement. At the top of the diagram the algorithm checks if the sum of power is less than the transformer power limit. If it is, each of the EV chargers additively increases their charge rate. They continue additively increasing at each time step until they receive a feedback signal from the transformer when it has become overloaded (the No case). The EV chargers then reduce their charge rate by a multiplicative factor. The control process continues until the battery is fully charged.

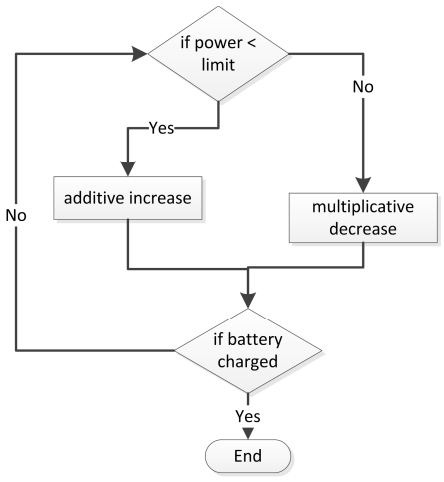

Figure 3. The basic AIMD algorithm.

It is a distributed algorithm that uses simple feedback control to modulate the power to the EV chargers. The algorithm minimises communication overhead (via one way broadcast) and can be operated at a frequent time rate (e.g. once per second). Their results show that the allocated power can track the available power very closely.

However, the algorithm uses a multiplicative decrease in charging rate that breaks the smooth charging constraint. For example, the charging rate could jump from $100 \%$ to $10 \%$ in one time step. This could lead to reduced lifetime of the battery. Also, the same as in the linear programming case, the solution is based on a variable rate EV charger. 


\section{Broadcast-Connection Rate}

Turitsyn et al. [9] present an interesting algorithm based on the EV chargers connecting with a given broadcast connection rate or probability. In this case, the EV charger used is a simple on-off charger. Fig. 4 shows an example profile of base load and an estimated power value $\mathrm{P}_{\text {excess }}$ that is the available power for charging of EVs during the night time period. With this estimate, the algorithm calculates the number of EVs that can charge in this night time period. The transformer broadcasts this connection rate percentage to the EV chargers. The EV chargers then attempt to connect with this probability. For example, $15 \%$ of the EV chargers will connect on full charge. The number of EVs available for charging is assumed known and fixed and once connected an EV will remain charging until its battery is full.

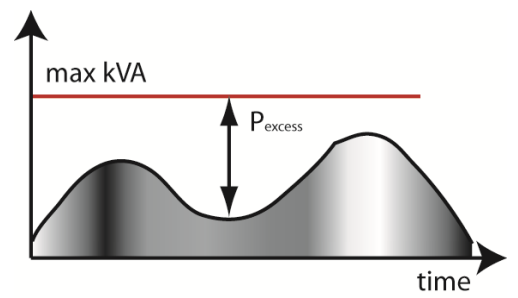

Figure 4. Pexcess in the night time period.

It is a simple distributed algorithm that only uses one-way communication and the simple on-off charger type. However, it makes several simplifying assumptions. It assumes that the night time base load is known from past records, that the number of available EVs is known and fixed, and that the EV charger size is known and the same for all the EVs. These assumptions are needed in order to calculate the connection rate percentage from the available power $\mathrm{P}_{\text {excess }}$. Also, the algorithm is not fair as once an EV connects, it will remain charging until its battery is full, thereby blocking other EVs from charging in the period.

However, despite these drawbacks the probability mechanism for controlling a given percentage of the EVs to connect is worth investigating further. We will use this idea in the second algorithm that we develop.

\section{ALGORITHM DESIGN}

\section{A. Variable Charging Rate Algorithm}

The variable charging rate algorithm uses the more sophisticated variable rate EV charger. It broadcasts from the transformer the charging rate $(0-100 \%)$ that each of the available EVs should charge at. The feedback is the measured power demand at the transformer. In our experiments we have chosen a broadcast frequency of once per minute as this is achievable for typical communication hardware such as power line communication (PLC).

Fig. 5 shows the simple control operation of the charging rate. If the power demand is less than the set point limit, then the charging rate is increased by one and if the demand is greater than the set point limit, then the charging rate is decreased by one. As the algorithm has a feedback mechanism there is no requirement to know the number of available EVs or their size of charge. The charging rate will adjust to the size of available power that it is controlling. For example, if a number of EVs leave to go to work, the charging rate will increase for the remaining EVs to take up the same amount of available power capacity.

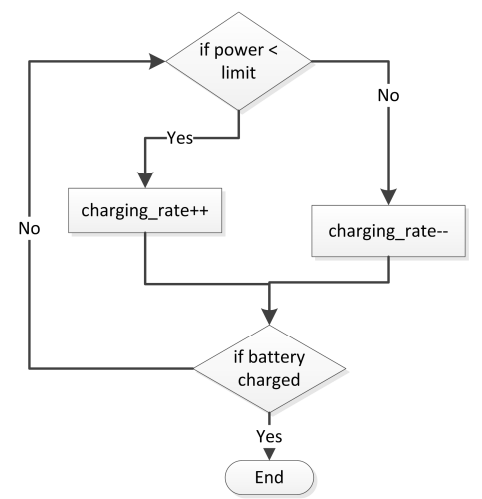

Figure 5. Variable charging rate algorithm.

This algorithm is similar to the AIMD approach. It has a higher communication overhead as it broadcasts at each minute interval. It fairly divides the available power among the EV chargers and it keeps within the constraints of the battery charger by only varying $1 \%$ per minute interval in both the increasing and decreasing directions. However, this algorithm uses the sophisticated variable rate charger. Next we outline a design that uses the simpler on-off charger.

\section{B. Variable Connection Rate Algorithm}

This algorithm is based on our previous algorithm and includes the use of probability to control the connection rate as in Turitsyn et al. [9]. In this algorithm the connection rate (0$100 \%$ ) is broadcast from the transformer at a frequency of once per minute, so each EV charger will attempt to connect once per minute with the given connection rate probability. The feedback of total power demand is measured at the transformer. The control operation is the same as shown in Fig. 5, except we are controlling a connection rate instead of a charging rate. If the total power demand is less than the set point limit, then the connection rate is increased by one, and if the demand is greater than the set point limit, then the connection rate is decreased by one.

The connection rate keeps increasing until the set point limit is reached and then it varies up and down to maintain the set point level. At the end of each minute interval, the EV chargers will again attempt to connect with the connection rate probability. The random process for connecting ensures that each of the EVs has a fair access to the available power. In essence, the EVs are multiplexed along the time domain in one minute intervals. Fig. 6 shows an example EV charger connecting (blue bar) with varying connection rate over time.

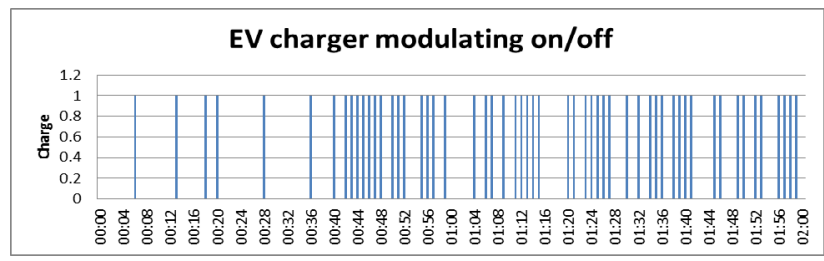

Figure 6. EV charger modulating on/off. 


\section{EXPERIMENTATION AND RESULTS}

A power system simulator, GridLab-D [10], was used to experiment with both algorithms. A test distribution system with one transformer feeding 90 houses within a neighbourhood was modelled. Each of the houses has one EV that has a routine of going from home to work and back again. Charging of the EVs only happens at home.

The base load for each of the houses is derived from measurements taken in Ireland during the Commission for Energy Regulation smart meter trial [2]. There is a separate base load for each of the 90 houses and the measurements are average power in kilo watts $(\mathrm{kW})$ in half hourly intervals. We interpolated this data to one minute periods in order to smooth the load profile so there are no sudden jumps in the demand (see Fig. 7). Having finer grained measured data of power demand would be preferable for testing purposes.

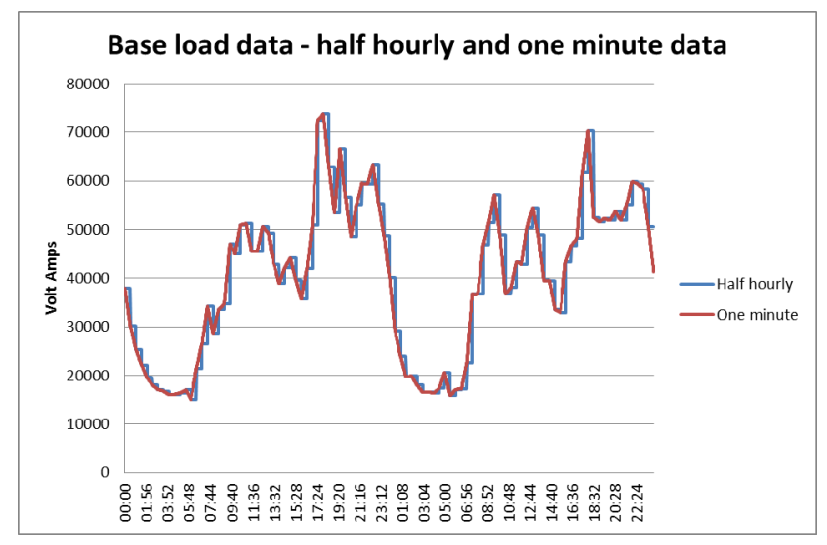

Figure 7. The interpolated one minute base load data.

\section{A. Variable Charging Rate Results}

Fig. 8 shows the algorithm operating over a 48 hour period. Initially the charging rate starts at zero and it must ramp up over a period of time. The EV chargers are sized at $1.32 \mathrm{~kW}$ each, so a $1 \%$ per minute increase is $13.2 \mathrm{~W} /$ minute for each available EV charger. If the $90 \mathrm{EVs}$ are available the ramp rate is $1.19 \mathrm{~kW} /$ minute.

The results show that the algorithm can closely follow the set point limit of 100 kilo Volt Amps (kVA). During the periods of tracking the set point, the mean aggregate demand is $99.8 \mathrm{kVA}$ and the standard deviation is $0.73 \mathrm{kVA}$. The control is based on the one minute averaged base load samples. We are assuming that variations to demand within this one minute period are short and are not a cause for concern for the transformer. If they were, the algorithm would have to set its set point below the maximum limit to account for the error.

For both of the days there is an overshoot. This occurs as the sharp rise in evening peak demand is coincident with the EVs arriving home from work. The controller is not fast enough to reduce the charging rate of the EVs from its $100 \%$ value (reached during off peak demand and few EVs available). Again, setting the set point below the max limit can address this over shoot in the controller.

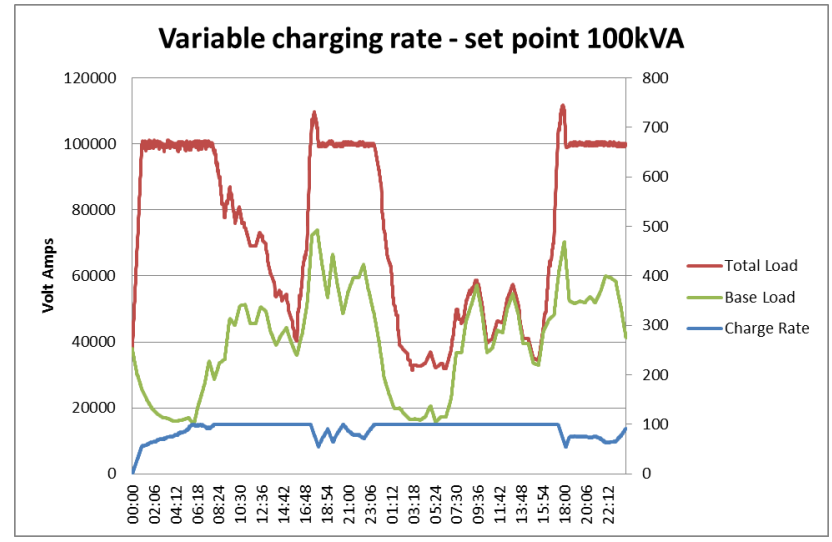

Figure 8. Variable charging rate at limit of transformer.

Fig. 9 shows the battery charge for each of the $90 \mathrm{EVs}$ over the two day period. The increasing sloping lines show the batteries gaining in charge and the horizontal lines occur when the battery has fully charged (at 1) or they have gone to work. The charge drops vertically when it returns from work. The figure shows that the EVs are charging at roughly the same rate as they have very similar slopes for their charging. This shows that the algorithm is fairly dividing out the available power to each of the EVs.

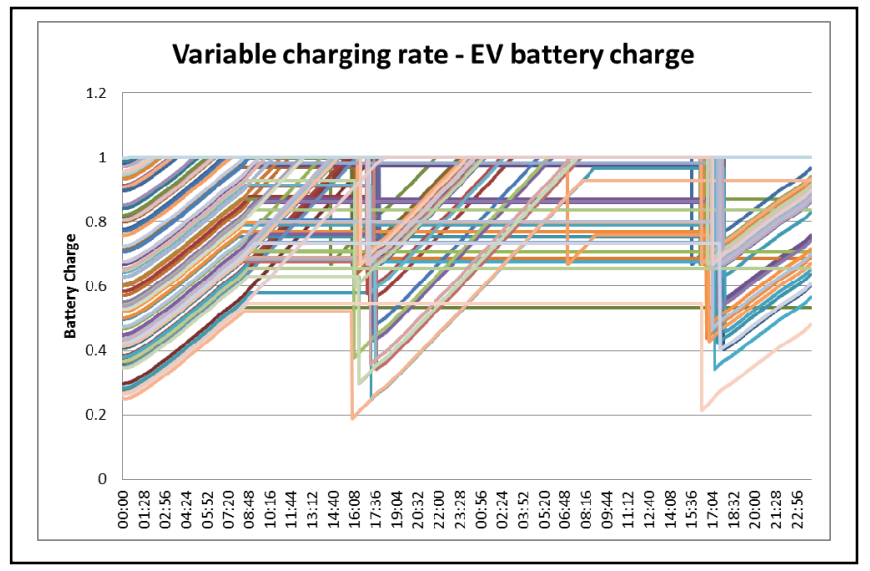

Figure 9. EV battery charge over time.

\section{B. Variable Connection Rate Results}

The variable connection rate results are similar to the charging rate results, but more erratic around the set point. The controller does not follow the set point limit as closely because of the random process for generating the probability to decide whether to connect or not. For example, the broadcast connection rate may be $75 \%$ but not exactly $75 \%$ of the EV chargers will connect due to the inherent error in the random probability process. This is shown in the results (see Fig. 10). 


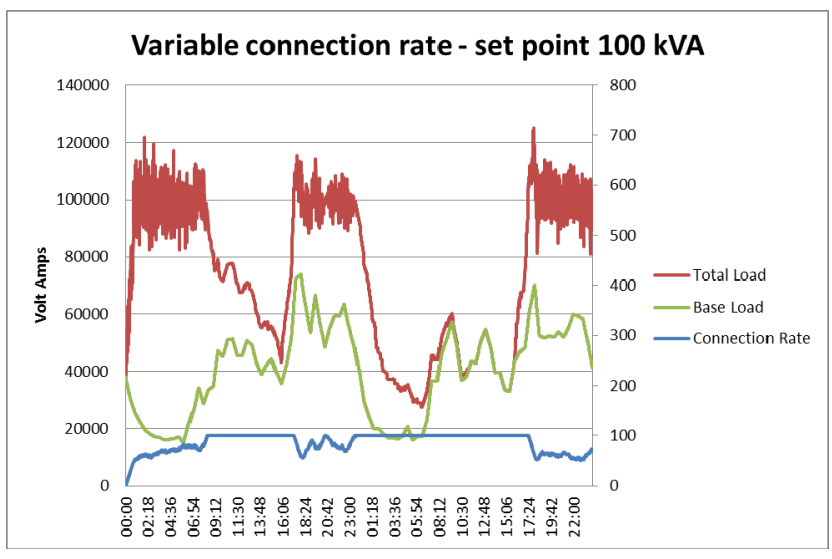

Figure 10. Variable connection rate at limit of transformer.

For these results, during the set point tracking periods, the mean was $99.2 \mathrm{kVA}$ and the standard deviation was 6.52 $\mathrm{kVA}$. The standard deviation is greater than in the variable rate control and the set point would have to be set below the limit by a greater margin. Also, as in the variable rate control, one can see the over shoot at the evening peak ramp ups. The controller is not fast enough to avoid this.

Fig. 11 shows the battery charge for each of the 90 EVs. The slopes of the EV charging lines are similar showing that the EVs are charging at roughly the same rate as each other. This shows that the algorithm is fairly dividing out the available power. This is the same result as in the variable charge rate algorithm, but in this case the algorithm is multiplexing the power across the one minute time intervals.

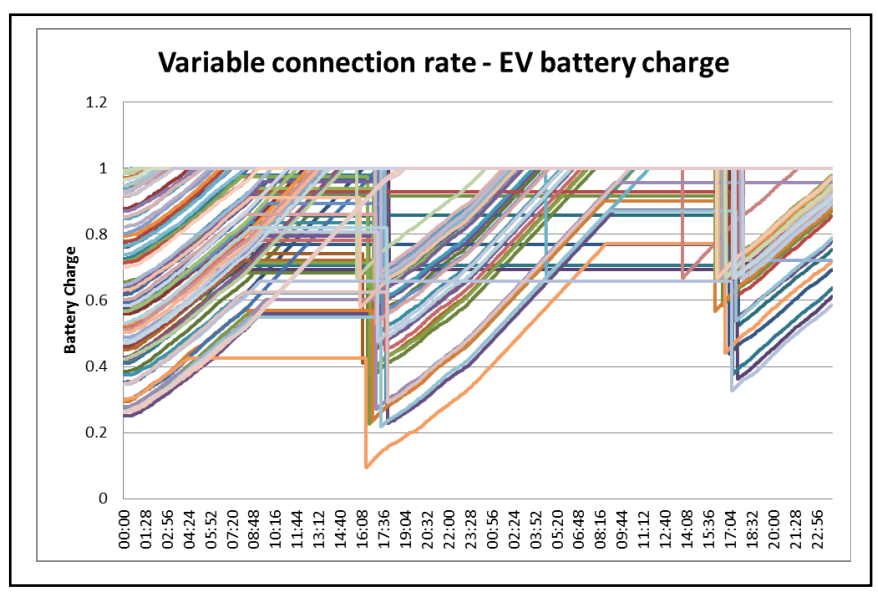

Figure 11. EV battery charge over time.

\section{CONCLUSIONS AND FUTURE WORK}

This paper proposes that demand is divided into two parts, a base load that can be influenced by for example, dynamic pricing, and a more tightly controlled flexible load, used to shape the aggregate demand. The paper looks at the scenario of coordinated charging of EVs as an example of this tightly controlled flexible load. Two algorithms are developed and simulated on a test distribution network. The first variable charging rate algorithm performs well by following closely its set point (mean $99.8 \mathrm{kVA}$ and standard deviation $0.73 \mathrm{kVA}$ ), but it relies on use of a sophisticated variable rate charger. The second variable connection rate algorithm uses probability to modulate the number of EVs connected over time. This algorithm uses the simpler on-off charger and achieves a similar performance (mean $99.2 \mathrm{kVA}$ and standard deviation $6.52 \mathrm{kVA}$ ), but with a greater error from the set point.

Future work will look at addition of electric storage heating and hot water heating into the network. We would also like to look at methods to coordinate the demand with periods of cheap renewable electricity supply. For example, we can schedule the set point level over time so that high demand coincides with periods of cheap supply. The problem now is ensuring that the utility of the appliances is maintained as they are now not set to charge as soon as possible.

\section{ACKNOWLEDGMENT}

This work was supported, in part, by Science Foundation Ireland grant 10/CE/I1855 to Lero - the Irish Software Engineering Research Centre (www.lero.ie).

\section{REFERENCES}

[1] Sustainable Energy Authority of Ireland. Smart Grid Roadmap. [Online]. Available: http://www.seai.ie/Publications/SEAI_Roadmaps/

[2] Commission for Energy Regulation. Smart meter trial data. [Online]. Available:

http://www.ucd.ie/issda/data/commissionforenergyregulationcer/

[3] Y. Cao, S. Tang, C. Li, P. Zhang, Y. Tan, Z. Zhang and J. Li. "An optimized EV charging model considering tou price and soc curve," IEEE Transactions on Smart Grid, 3 (1), pp. 388-393, 2012.

[4] K. Clement-Nyns, E. Haesen and J. Driesen. "The impact of charging plug-in hybrid electric vehicles on a residential distribution grid," IEEE Transactions on Power Systems, 25 (1), pp. 371-380, 2010.

[5] S. Deilami, A. Masoum, P. Moses and M. Masoum. "Real-time coordination of plug-in electric vehicle charging in smart grids to minimize power losses and improve voltage profile," IEEE Transactions on Smart Grid, 2 (3), pp. 456-467, 2011.

[6] M. Erol-Kantarci and H. Mouftah. "Prediction-based charging of phevs from the smart grid with dynamic pricing," in Proc. 2010 IEEE 35th Conference on Local Computer Networks (LCN) pp. 1032-1039, Denver, Colorado, U.S.A.

[7] P. Richardson, D. Flynn, and A. Keane. "Optimal charging of electric vehicles in low-voltage distribution systems," IEEE Transactions on Power Systems, 27 (1), pp. 268-279, 2012.

[8] S. Stüdli, E. Crisostomi, R. Middleton and R. Shorten. "A flexible distributed framework for realising electric and plug-in hybrid vehicle charging policies," International Journal of Control, 85 (8), pp. 1130$1145,2012$.

[9] K. Turitsyn, N. Sinitsyn, S. Backhaus, and M. Chertkov. "Robust broadcast-communication control of electric vehicle charging," in Proc. 2010 First IEEE International Conference on Smart Grid Communications (SmartGridComm) pp. 203-207, Gaithersburg, MD, USA.

[10] U.S. Department Of Energy. Gridlab-D, [Online]. Available: http://www.gridlabd.org/ 